\title{
Conhecimento popular: impactos e métodos de controle de Achatina fulica em Valença - RJ, Brasil
}

\author{
Evelyn Durço ${ }^{1,4 *}$ \\ Tércia Vargas ${ }^{1,4}$ \\ Lidiane Silva ${ }^{2,4}$ \\ Vinícius Marins Carraro ${ }^{3}$ \\ ${ }^{1}$ PPG em Comportamento e Biologia Animal \\ Universidade Federal de Juiz de Fora, Juiz de Fora - MG, Brasil \\ ${ }^{2}$ PPG em Ciências Veterinárias \\ Universidade Federal Rural do Rio de Janeiro, Rio de Janeiro - RJ, Brasil \\ ${ }_{3}^{3}$ Centro de Ciências da Saúde, Universidade Severino Sombra,Vassouras - RJ, Brasil \\ Graduação em Ciências Biológicas - Centro Universitário Geraldo Di Biase, Volta Redonda - RJ, Brasil \\ ${ }^{4}$ Museu de Malacologia Prof. Maury Pinto de Oliveira, Instituto de Ciências Biológicas \\ Universidade Federal de Juiz de Fora, CEP 36036-900, Juiz de Fora - MG, Brasil \\ * Autor para correspondência \\ evelynbiobacharel@gmail.com
}

Submetido em 22/03/2012

Aceito para publicação em 06/12/2012

\section{Resumo}

O objetivo deste estudo foi verificar a incidência do caramujo africano Achatina fulica no bairro Cambota, Valença-RJ, Brasil, e investigar as estratégias de controle adotadas pela população. Questionários epidemiológicos aplicados a 105 moradores averiguaram a existência de contato deles com o animal, o risco de contágio por parasitos, por conta dos hábitos de higiene, e os métodos de controle adotados. A presença dos moluscos foi relatada em 52,5\% das residências visitadas. Dessas, 51,4\% apresentaram roedores. Moluscos coletados foram analisados quanto à presença de nematoides. Nas residências positivas para a presença de $A$. fulica foi relatado contato direto com os moluscos $(21,9 \%)$ por manuseio (muitas vezes inadequado) ou por ingestão. Todos os entrevistados disseram utilizar alguma técnica para higienização dos alimentos e 67,6\% relataram conhecer a angiostrongilíase. Todos os entrevistados disseram praticar o extermínio dos moluscos, $28,5 \%$ desses efetuando a quebra da concha. Apesar da alta incidência de A. fulica, não foram encontradas larvas de Angiostrongylus sp. ou de outros nematoides de importância médico-veterinária nos espécimes analisados.

Palavras-chave: Controle; Levantamento epidemiológico; Molusco terrestre

\section{Abstract}

Popular knowledge: impacts and methods for controlling Achatina fulica in Valença - RJ, Brazil. This study aimed to check the incidence of the African snail Achatina fulica in the Cambota neighborhood, Valença, Rio de Janeiro, Brazil, and investigate control strategies adopted by the population. Epidemiological questionnaires applied to 105 inhabitants evaluated the existence of their contact to the animal, the risk of 
infection by parasites, due to hygiene behaviors, and the control methods adopted. The presence of mollusks was reported in $52.5 \%$ of visited households. Out of these, $51.4 \%$ had rodents. Collected mollusks were analyzed with regard to the presence of nematodes. In the households with a positive result for the presence of $A$. fulica, direct contact to the mollusks was reported (21.9\%) by handling (often inappropriate) or intake. All respondents said to use some technique for food hygiene and $67.6 \%$ said to know angiostrongyliasis. All respondents said to practice extermination of mollusks, $28.5 \%$ of them by breaking the shell. Despite the high incidence of $A$. fulica, larvae of Angiostrongylus sp. or other nematodes of medico-veterinary importance weren't found in the analyzed specimens.

Key words: Control; Epidemiological survey; Infection; Terrestrial mollusk

\section{Introdução}

A introdução de espécies exóticas é considerada a segunda maior causa de extinção de espécies no planeta, o que afeta diretamente a biodiversidade (CORADIN; TORTATO, 2006). A espécie Achatina fulica Bowdich, 1822 (Achatinidae) popularmente conhecida como caramujo africano, é um molusco terrestre, nativo da região leste-nordeste da África (VASCONCELLOS; PILE, 2001), e é reconhecida como uma das cem piores espécies invasoras no mundo (ALOWE et al., 2004).

Tal espécie foi introduzida ilegalmente no Brasil com o objetivo de comercialização, mas a não aceitação pelo mercado resultou no abandono das criações de maneira inadvertida, iniciando-se assim a sua proliferação (THIENGO et al., 2007). A resistência a condições ambientais adversas e o elevado potencial reprodutivo, característicos da espécie, culminaram na sua rápida dispersão (SIMIÃO; FISHER, 2004).

A significativa ocupação de ambientes antrópicos pelo caramujo africano é preocupante visto que esses animais podem atuar na transmissão de graves doenças para humanos e outros animais, como a angiostrogilíase, helmintose provocada por espécies do gênero Angyostrongylus Kamensky, 1905 (SIMIÃO; FISHER, 2004; CALDEIRA et al., 2007; THIENGO, 2007). Tais espécies utilizam o molusco como hospedeiro intermediário, com a infecção ocorrendo pela ingestão de larvas de primeiro estádio (L1) nas fezes de roedores,e que se desenvolvem até o terceiro estádio (L3) (TELES et al., 1997; GRAEFF-TEIXEIRA, 2007). Esses parasitos têm como hospedeiro definitivo natural roedores como Rattus norvegicus Berkenhout, 1769 e
Rattus rattus Linnaeus, 1758, que se infectam através da ingestão do molusco, vegetais ou água contaminada contendo a larva de terceiro estádio (L3) (DIAZ, 2008; LV et al., 2009; FORONDA et al., 2010). A infecção para o homem (hospedeiro acidental) também se dá através de larvas de terceiro estádio (L3), que podem ser encontradas em alimentos crus e mal-cozidos ou através da própria ingestão do molusco (TELES et al., 1997). Além disso, A. fulica também pode atuar como vetor mecânico para outras enfermidades até então desconhecidas, evidenciada pela elevada carga parasitária do molusco (THIENGO et al., 2005; CORADIN; TORTATO, 2006).

Para a elaboração de estratégias eficientes de controle de A. fulica é importante o envolvimento das comunidades humanas afetadas pela presença do animal. Assim, é necessário investigar o conhecimento da população afetada sobre a proliferação do molusco e estratégias de controle utilizadas (SOUZA et al., 2007).

O caramujo africano encontra-se amplamente distribuído no estado do Rio de Janeiro podendo ser encontrado em quase todos os municípios desse estado, incluindo o município de Valença (ZANOL et al., 2010).

Foram objetivos desse trabalho: (i) verificar a incidência do caramujo africano no Bairro Cambota e o conhecimento da população sobre a temática abordada enfocando os meios de controle e prevenção utilizados em uma comunidade invadida pelo molusco exótico através da análise etimológica e (ii) verificar a presença de nematoides de importância médico-veterinária nos moluscos. 


\section{Material e Métodos}

Para a obtenção de um perfil de infestação, foram visitadas aleatoriamente, 200 residências em todas as ruas pertencentes ao Bairro Cambota, no Município de Valença, Rio de Janeiro, Brasil. O bairro foi escolhido devido ao maior número de reclamações recebidas através da Secretaria Municipal de Saúde de Valença, e se caracteriza como uma área periférica, de infra-estrutura deficiente, com muitas ruas sem asfalto ou saneamento básico e com muitos terrenos baldios.

Nas residências onde se verificou a presença do molusco foi aplicado um questionário de múltipla escolha (Anexo 1) para avaliar o conhecimento dos moradores sobre a presença do animal; se havia contato direto com este e como era feito; forma de manuseio utilizada; métodos usados na higienização de verduras, legumes e frutas; nível de informação sobre a angiostrongilíase; existência de roedores na residência; e a utilização de algum método para eliminação dos moluscos.

Foram coletados 70 espécimes em pontos aleatórios do bairro, que foram acondicionados adequadamente e enviados através da Secretaria de Saúde Municipal de Valença ao Laboratório de Referência Nacional em Malacologia Médica do Instituto Oswaldo Cruz, Rio de Janeiro, RJ, onde foram analisados quanto à presença de larvas de Angiostrongylus sp. ou outros nematoides, através do método de digestão artificial modificada (THIENGO et al., 2008).

\section{Resultados e Discussão}

Das 200 residências visitadas, a presença de $A$. fulica foi registrada em 105 (52,5\%) destas, nas quais as entrevistas foram realizadas (um entrevistado/ residência). Os resultados da entrevista podem ser observados na Tabela 1.

O questionário epidemiológico revelou que 21,9\% dos moradores entrevistados tiveram contato direto com o molusco. Dentre os que tiveram contato, $98,9 \%$ somente manusearam os moluscos e um morador relatou ter ingerido os animais. Dentre os indivíduos que relataram ter manuseado os animais, $86,5 \%$ relataram o uso de algum tipo de proteção. Todos os entrevistados disseram que realizam alguma forma de higienização dos alimentos (Figura 1).

TABELA 1: Questionário aplicado aos moradores de 105 residências do bairro Cambota, município de Valença, Rio de Janeiro onde se verificou a presença do caramujo africano Achatina fulica.

\begin{tabular}{lcc}
\hline & \multicolumn{2}{c}{$\begin{array}{c}\text { Frequência } \\
\text { relativa }(\%)\end{array}$} \\
\cline { 2 - 3 } & Sim & Não \\
\hline Contato direto com o animal & 21,9 & 78,1 \\
Manuseio do animal & 98,9 & 1,1 \\
$\quad$ Manuseio com proteção & 86,5 & 13,5 \\
Ingestão do animal & 1,1 & 98,9 \\
Higienização do alimento & 100,0 & 0,0 \\
Conhecimento da transmissão da & 67,6 & 32,4 \\
angiostrongilíase & 51,4 & 58,6 \\
Presença de roedores na residência & 100,0 & 0,0 \\
Realiza controle dos moluscos & 28,5 & 71,5 \\
Quebra das conchas & \multicolumn{2}{c}{} \\
\hline
\end{tabular}

FIGURA 1: Percentual de métodos de higienização dos alimentos utilizados pelos moradores de residências com infestação do caramujo africano (Achatina fulica) no Bairro Cambota, Município de Valença, RJ.

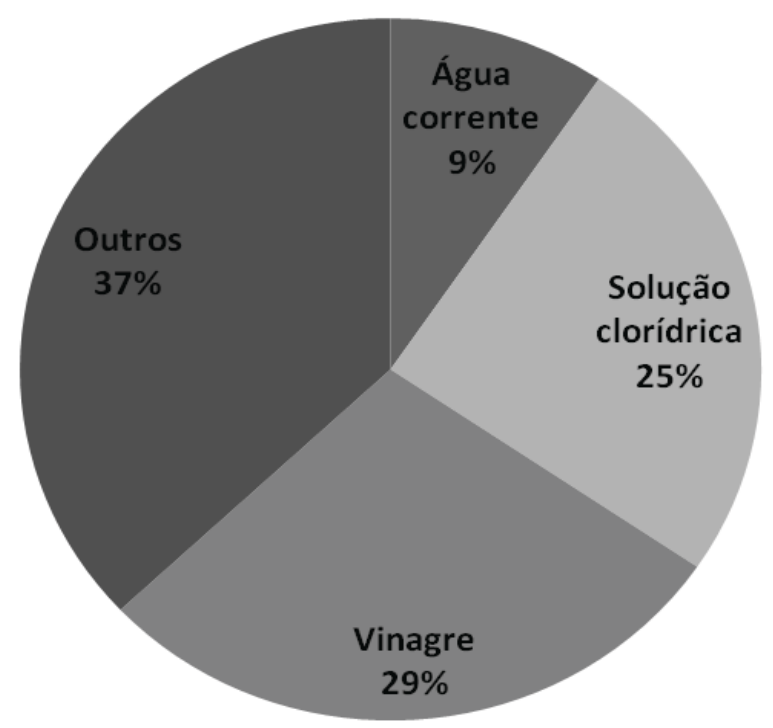

A infecção humana pelos parasitos do gênero Angyostrongylus geralmente é acidental através da ingestão de seus hospedeiros intermediários ou paratênicos. Além disso, pode ocorrer infecção através da ingestão de alimentos contaminados com muco do molusco contendo a larva de terceiro estágio do parasito 
(GRAEFF-TEIXEIRA, 2007). Dentre os métodos de higienização dos alimentos, verificou-se o uso de água corrente, vinagre, solução clorídrica e outros. Dentro da classe outros, observa-se o uso de sabão de côco, detergente e limão. Porém ressalta-se que o único meio correto e eficaz para a assepsia destes é o uso de solução clorídrica diluída em água segundo recomendação do Ministério do Meio Ambiente (MMA) (BRASIL, 2008), o qual é utilizado apenas por $25 \%$ dos entrevistados.

A maioria dos entrevistados mostrou ter conhecimento sobre a angiostrongilíase (67,6\%). Simião e Fisher (2004) observaram em Pontal, Paraná, que os entrevistados não mostraram conhecimento da doença, relacionando o molusco a outras enfermidades como meningite, leishmaniose, alergias e doenças de pele. Colley e Fisher (2009) também observaram que nenhum dos entrevistados soube relacionar os verdadeiros problemas de saúde ocasionados pelo caramujo invasor, sendo citados: meningite, queimaduras, problemas respiratórios, cegueira, barriga d'água, câncer, manchas de pele, coceiras, febre, feridas e vômitos. Muito embora pesquisas apontem uma rejeição ao molusco por parte da população devido à possibilidade de transmissão de doenças, em geral os entrevistados não têm conhecimento sobre o ciclo de tais doenças (PEREIRA, 2010). A compreensão de doenças como a angiostrongilíase é muito importante, uma vez que podem estar sendo sub-diagnosticadas (GRAEFFTEIXEIRA, 2007). O único tratamento indicado para esta zoonose, até o momento, é a intervenção cirúrgica. O uso de anti-helmínticos não é indicado, induzindo a migração errática do parasito, agravando o quadro patológico(MORERA; BONTEMPO, 1985).

Foi verificada a presença de roedores em 51,4\% das residências positivas para a presença de $A$. fulica. A presença de roedores é relevante, pois esses atuam naturalmente como hospedeiros definitivos do Angiostrongylus costaricensis (Morera \& Céspedes, 1971) e Angiostrongilus cantonensis (Chen, 1985). Desse modo, podem atuar com reservatórios do parasito e como uma fonte de infecção para o molusco (BONETTI; GRAEFF-TEIXEIRA, 1998; MOTA; LENZI, 2005). Segundo Colley e Fisher (2009), devido à sua grande população, $A$. fulica torna-se uma fonte de alimento em excesso para alguns animais que, por consequência, também pode aumentar muito a população de seus principais predadores, os roedores.

Sobre o controle de $A$. fulica, constatou-se que $100 \%$ dos entrevistados praticam algum método de controle, cujos percentuais são demonstrados na Figura 2. As substâncias mais utilizadas foram sal de cozinha e cal virgem para matar os animais, seguidos por incineração e/ou enterramento dos moluscos. Esses dados corroboram os encontrados por Junior e Nunes (2009) em uma área de proteção ambiental em Várzea Grande, Mato Grosso. Fisher e Colley (2005) também registraram dados semelhantes no litoral do Paraná.

FIGURA 2: Percentual de métodos utilizados pelos entrevistados para o extermínio de Achatina fulica no Bairro Cambota, Município de Valença, RJ.

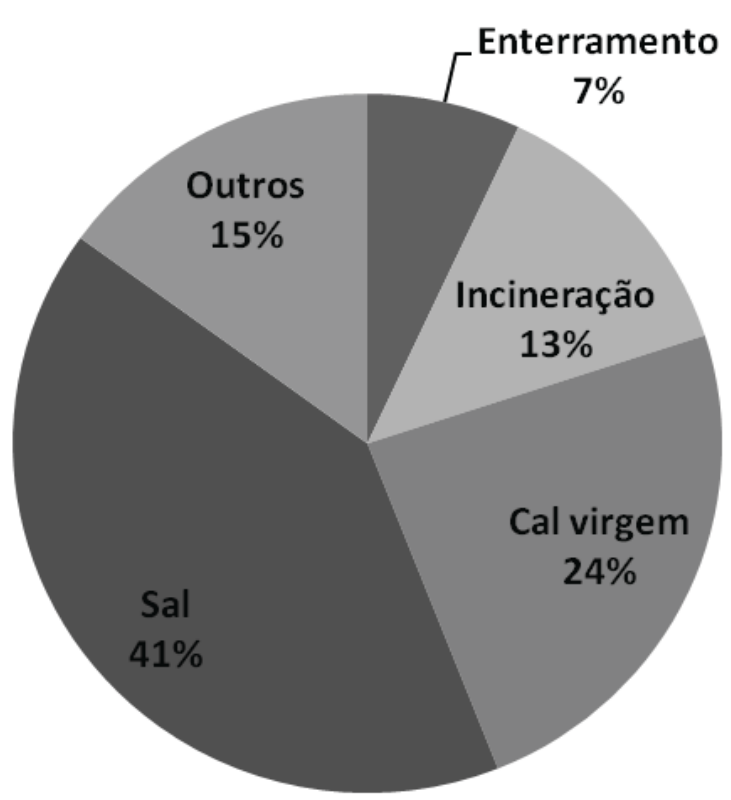

Atualmente o controle de A. fulica realizado no Brasil baseia-se nas Instruções Normativas do Ibama no ${ }^{-} 73$, de 18 de agosto de 2005 (BRASIL, 2005) e $\mathrm{n}^{-}$ 109, de 3 de agosto de 2006 (BRASIL, 2006). Segundo estas instruções, o procedimento de controle do molusco consiste primeiramente na identificação da espécie em suspeita, evitando o extermínio de moluscos nativos. Após a identificação deve-se realizar o controle através da coleta manual do animal e de seus ovos, utilizando luvas e recipientes plásticos. Os moluscos devem ser incinerados. Orienta-se a não utilização de 
sal, moluscicidas ou outros produtos tóxicos a outros animais.

Métodos inadequados de descarte dos moluscos foram registrados como junto ao lixo doméstico ou em recursos hídricos. O descarte junto ao lixo doméstico e em recursos hídricos traz grande preocupação, podendo colaborar para dispersão do animal (SIMIÃO; FISHER, 2004; FISHER, 2009). Foi relatado também o uso do molusco na alimentação de aves domésticas e o esmagamento do animal.

Quando questionados sobre a quebra da concha do molusco após o extermínio, verificou-se que apenas $28,5 \%$ faziam uso dessa prática. A quebra da concha do molusco é importante, pois essa pode acumular água da chuva, tornando-se um possível criadouro de dípteros e servindo como abrigo para jovens de $A$. fulica recémeclodidos, o que foi inclusive observado no presente estudo durante as coletas.

Ainda não existem métodos eficazes para o controle do molusco no Brasil, merecendo grande atenção das autoridades competentes (JUNIOR; NUNES, 2009). Segundo Colley e Fisher (2009), as ações de controle e manejo de $A$. fulica no país não têm contribuído para a diminuição de suas populações. $\mathrm{O}$ controle manual está baseado praticamente na catação e posterior eliminação do animal, porém sua eficiência depende da combinação de várias outras medidas(COLLEY, 2010). Segundo Oliveira et al. (2010), são necessárias ações de conscientização e educação ambiental. Há uma grande necessidade no controle de $A$. fulica, cuja situação no país foi de uma explosão populacional descontrolada cerca de dez anos após sua introdução, podendo acarretar no aumento das doenças que tenham essa espécie como transmissor(GRAEFF-TEIXEIRA, 2007). Tal fato tem sido observado no Estado do Rio de Janeiro, onde o número de municípios com registros de ocorrência de $A$. fulica aumentou rapidamente, de 2002 a 2009 (ZANOL et al., 2010), sendo registrados novos parasitos de importância epidemiológica (THIENGO, 2007).

Não foram encontradas larvas de Angiostrongylus sp. ou de outros nematóides de importância médicoveterinária nos espécimes coletados e analisados no presente estudo. Carvalho et al. (2003) sugerem que $A$. fulica apresenta baixo potencial de transmissão para angiostrongilíase. Porém, um estudo epidemiológico realizado em Pernambuco mostrou sua participação ativa na transmissão da doença (THIENGO et al., 2010). Aponta-se também um risco potencial de A. fulica participar do ciclo desses nematóides tendo em vista a baixa especificidade de hospedeiros intermediários (THIENGO et al., 2005), destacando-se a existência de registros de dois casos de meningite eosinofílica no estado do Espírito Santo (CALDEIRA et al., 2007) e um em Pernambuco (LIMA et al., 2009). Nestes casos, no entanto, não foi confirmada a participação de $A$. fulica nas infecções.

Apesar de não verificada a infecção dos moluscos por parasitos de importância médico-veterinária no presente estudo, tal fato já foi relatado no município de Valença-RJ para Strongyluris-like, Aelurostrongylus abstrusus (Railliet, 1898), Rhabditis sp. e outros parasitos (THIENGO et al., 2007). Sugere-se que sejam realizadas pesquisas adicionais periódicas no município em questão, a fim de obter um perfil de infestação de A. fulica e de infecção dos moluscos por nematoides. A avaliação do conhecimento da população torna-se importante por contribuir para um programa de controle mais eficaz e que atenda a realidade do município.

\section{Agradecimentos}

À Secretaria Municipal de Saúde de Valença e ao Laboratório de Referência Nacional em Malacologia Médica do Instituto Oswaldo Cruz.

\section{Referências}

ALOWE, S.; BROWNE, M.; BOUDJELAS, S. 100 of the world's worst invasive alien species. A selection from the global invasive species database. 2004. Disponível em: <www.issg.org/database>. Acesso em: 21 set. 2010.

BONETTI, V.C. B. D. O.; GRAEFF-TEIXEIRA, C. Angiostrongylus costaricensis and the intermediate hosts: observations on elimination of L3 in the mucus and inoculation of L1 through the tegument of molluscs. Revista da Sociedade Brasileira de Medicina Tropical, Uberaba, v. 31, n. 3, p. 289-294, 1998.

BRASIL. Instrução Normativa no $\mathbf{7 3}$, de 18 de agosto de 2005. 2005. Disponível em: <www.cetesb.sp.gov.br/licenciamento/ legislaçãofederal/inst_normativa/2005_instr_norm_IBAMA>. Acesso em: 21 set. 2010. 
BRASIL. Instituto Brasileiro do Meio Ambiente e dos Recursos Naturais Renováveis - Parecer Técnico no 006/03- GGFAUBrasília. Brasília: IBAMA, 2006. 12 p.

BRASIL. Ministério da Saúde. Vigilância e Controle de Moluscos de Importância Epidemiologia. Série A Normas e Manuais Técnico. 2. ed., Brasília: Ed. MS, 2008. 345 p.

CALDEIRA, R. L.; MENDONÇA, C. L.; GOVEIA, C. O.; LENZI, H. L.; GRAEFF-TEIXEIRA, C.; LIMA, W. S.; MOTA, E. M; PECORA, I. L.; MEDEIROS, A. M. Z.; CARVALHO, O. S. First record of molluscs naturally infected with Angiostrongylus cantonensis (Chen, 1935) (Nematoda: Metastrongylidae) in Brazil. Memórias do Instituto Oswaldo Cruz, Rio de Janeiro, v. 102, n. 7, p. 887-889, 2007.

CARVAlHO, O. S.; TELES, H. M. S.; MOTA, E. M.; MENDONÇA, C. L.; LENZI, H. L. Potencialidade de Achatina fulica Bowdich, 1822 (Mollusca: Gastropoda), como hospedeiro intermediário potencial de Angiostrongylus costaricensis Morera e Céspedes, 1971. Sociedade Brasileira de Medicina Tropical, Uberaba, v. 36, n. 6, p. 743-745, 2003.

CARVALHO JUNIOR, V. C. B.; NUNES, J. R. S. Ocorrência e distribuição do caramujo africano "Achatina fulica" Bowdich, 1822, no município de Várzea Grande-MT. Revista de Engenharia Ambiental, Espírito Santo do Pinhal, v. 6, n. 2, p. 606-620, 2009.

COLLEY, E. Medidas de controle de Achatina fulica. In: FISCHER, M. L.; COSTA, L. C. M. (Ed.). O caramujo gigante africano Achatina fulica no Brasil. Curitiba: Champagnat Editora - PUCPR, 2010. p. 203-229.

COLLEY, E.; FISHER, M. L. Avaliação dos problemas enfrentados no manejo do caramujo gigante africano Achatina fulica (Gastropoda: Pulmonata) no Brasil. Revista Brasileira de Zoologia, Curitiba, v. 26, n. 4, p. 674-683, 2009.

CORADIN, L.; TORTATO, D. T. Espécies exóticas invasoras: situação brasileira. Brasília: Ministério do Meio Ambiente, Secretaria de Biodiversidade e Florestas, 2006. 24 p.

DIAZ, J. H. Helminth eosinophilic meningitis: emerging zoonotic diseases in the South. Journal of the Louisiana State Medical Society, Los Angeles, v. 160, p. 333-342, 2008.

FISHER, M. L. Reactions of the invasive alien species Achatina fulica to abiotic factors: perspectives for the management. Revista Brasileira de Zoologia, Curitiba, v. 26, n. 3, p. 379-385, 2009.

FISHER, M. L.; COLLEY, E. Espécies invasoras em reservas naturais: caracterização da população de Achatina fulica Bowdich, 1822 na Ilha Rasa, Guaraqueçaba, Paraná. Biota Neotropica, Campinas, v. 5, n. 1, p. 1-17, 2005.

FORONDA, P.; LÓPEZ-GONZÁLEZ, M.; MIGUEL, J.; TORRES, J.; SEGOVIA, M.; ABREU-ACOSTA, N.; CASANOVA, J. C.; VALLADARES, B.; MAS-COMA, S.; BARGUES, M. D.; FELIU, C. Finding of Parastrongylus cantonensis (Chen, 1935) in Rattus rattus in Tenerife, Canary Islands (Spain). Acta Tropica, Miami, v. 114, p. 123-127, 2010.

GRAEFF-TEIXEIRA, C. Expansion of Achatina fulica in Brazil and potential increased risk for angiostrongyliasis. Transactions of the Royal Society of Tropical Medicine and Hygiene, London, v. 101, p. 743-744, 2007.

LIMA, A. R. M. C.; MESQUITA, S. D.; SANTOS, S. S.; AQUINO, E. R. P. D.; ROSA, L. R. S.; DUARTE, F. S.; TEIXEIRA, A. O.; COSTA, Z. R. S.; FERREIRA, M. L. B. Alicata disease: neuroinfestation by Angiostrongylus cantonensis in Recife, Pernambuco, Brasil. Arquivos de Neuro-Psiquiatria, São Paulo, v. 67, n. 4, p. 1093-1096, 2009.

LV, S.; ZHANG, Y.; LIU, H. X.; ZHANG, C. W.; STEINMANN, P.; ZHOU, X. N.; UTZINGER, J. Angiostrongylus cantonensis: morphological and behavioral investigation within the freshwater snail Pomacea canaliculata. Parasitology Research, Heidelberg, v. 104, p. 1351-1359, 2009.

MORERA, P.; BONTEMPO, I. Accion de algunos antihelminticos sobre Angiostrongylus costaricensis. Revista Médica del Hospital Nacional de Niños Dr. Carlos Sáenz Herrera, San José, v. 20, n. 2, p. 165-174, 1985.

MOTA, E. M.; LENZI, H. L. Angiostrongylus costaricensis: complete redescription of the migratory pathways based on experimental Sigmodonhispidus infection. Memórias do Instituto Oswaldo Cruz, Rio de Janeiro, v. 100, n. 4, p. 407-420, 2005.

OLIVEIRA, A. P. M.; TORRES, E. J. L.; MALDONADO, A.; ARAÚJO, J. L. B.; FERNANDEZ, M. A.; THIENGO, S. C. Achatina fulica como hospedeiro intermediário de nematódeos de interressse médico-veterinário em Goiás, Brasil. Revista de Patologia Tropical, Goiânia, v. 39, n. 3, p. 199-210, 2010.

PEREIRA, Z. M. Estudo das percepções de estudantes da rede pública e da helmintofauna associada ao caramujo africano Achatina fulica Bowdich, 1822 (Mollusca, Gastropoda) em Barra do Piraí (RJ): subsídios para uma intervenção educativa. 2010. 115 f. Dissertação (Mestrado em Ensino em Biociências e Saúde) - Instituto Oswaldo Cruz, Rio de Janeiro. 2010.

SIMIÃO, M. S.; FISHER, M. L. Estimativa e inferência do método de controle do molusco exótico Achatina fulica Bowdich, 1822 (Stylommatophora; Achatinidae) em Pontal do Paraná, litoral do Estado do Paraná. Cadernos de Biodiversidade, Curitiba, v. 4, p. 74-83, 2004.

SOUZA, R. M.; ALVES, A. G. C.; ALVEZ, M. Z. Conhecimento sobre o molusco gigante africano Achatina fulica entre estudantes de uma escola pública na Região Metropolitana de Recife. Biotemas, Florianópolis, v. 20, p. 81-89, 2007.

TELES, H.; VAZ, J.; FONTES, L.; DOMINGOS, M. Ocorrência de Achatina fulica Bowdich, 1822 (Mollusca, Gastropoda) no Brasil: caramujo hospedeiro intermediário de Angiostrongilíases. Revista de Saúde Pública, São Paulo, v.31, p.310-2, 1997.

THIENGO, S. C. Helmintoses de interesse médico-veterinário transmitidas por moluscos no Brasil. In: ENCONTRO BRASILEIRO DE MALACOLOGIA, XVIII, 2007, Rio de Janeiro. Anais... Rio de Janeiro: SBM, 2007. p. 287-294.

THIENGO, S. C.; BARBOSA, A. F.; COElHO, P. M.; FERNANDEZ, M. A. Moluscos exóticos com importância médica no Brasil. In: SIMPÓSIO BRASILEIRO SOBRE ESPÉCIES EXÓTICAS INVASORAS, I, 2005, Brasília, Anais. Brasília: SBEEI. Disponível em: <http://www.mma.gov.br/invasoras $>$. Acesso em: 21 setembro 2010.

THIENGO, S. C.; FARACO, F. A.; SALGADO, N. C.; COWIE, R. H.; FERNANDEZ, M. A. Rapid spread of an invasive snail in South America: the giant African snail, Achatina fulica, in Brazil. Biological Invasions, Dordrecht, v. 4, n. 1, p. 1-10, 2007.

THIENGO, S. C.; FERNANDEZ, M. A. Achatina fulica: um problema de saúde pública? In: FISCHER, M. L.; COSTA, L. C. M. (Eds). O caramujo gigante africano Achatina fulica no Brasil. 
Curitiba: Champagnat Editora - PUCPR, 2010. p. 189-202.

THIENGO, S. C.; FERNANDEZ, M. A.; TORRES, E. J. L.; COELHO, P.M.; LANFREDI, R. M. First record of a nematode Metastrongyloidea (Aelurostrongylus abstrusus larvae) in Achatina (Lissachatina) fulica (Mollusca, Achatinidae) in Brazil. Journal of Invertebrate Pathology, Riverside, v. 98, p. 34-39, 2008.

VASCONCELLOS, M. C.; PILE, E. Ocorrência de Achatina fulica no Vale do Paraíba, Estado do Rio de Janeiro, Brasil. Revista de Saúde Pública, São Paulo, v. 35, n. 6, p. 582-584, 2001.

ZANOL, J.; FERNANDEZ, M. A.; OLIVEIRA, A. P. M.; RUSSO, C. A. M.; THIENGO, S.C. O caramujo exótico invasor Achatina fulica (Stylommatophora, Mollusca) no Estado do Rio de Janeiro (Brasil): situação atual. Biota Neotropica, Campinas, v. 10, n. 3, p. $67-78,2010$. 


\section{Anexo 1}

\section{Questionário Epidemiológico}

\section{Conhecimento popular: impactos e}

métodos de controle de Achatina fulicano

Bairro Cambota, Valença - RJ, Brasil

1) Já teve um contato direto com o caramujo africano?

$$
\text { [.. }] \operatorname{sim} \quad[. .] \text { não }
$$

2) Se marcado SIM, de que forma se deu o contato direto com animal?

[..] ingestão [..] contato manual ou com outras partes do corpo

3) Qual a forma de manuseio utilizado?

$$
\text { [..] com proteção [..] sem proteção }
$$

4) Realiza a higienização dos alimentos (verduras, frutas e legumes)?

[..] $\operatorname{sim} \quad$ [.. $]$ não

5) Se sim, que tipo de higienização?

[..] água corrente [..] solução clorídrica

[..] vinagre [..] outros. Quais:

6) Possui conhecimento sobre a transmissão da angiostrongilíase?
[..] $\operatorname{sim} \quad[.$.$] não$

7) Já observou a presença de roedores (ratos) na residência?

[..] $\operatorname{sim} \quad[.$.$] não$

8) Realiza algum tipo de controle do caramujo africano?

[..] $\operatorname{sim} \quad[.$.$] não$

9) Qual tipo de controle do caramujo realizado?

[..] sal

[..] cal virgem

[..] enterrar

[..] incinerar

[..] outros. Quais:

10) Após o controle do molusco ou ao observar conchas vazias pratica a quebra destas conchas?
[..] $\operatorname{sim}$
[..] não 